\title{
Modulation of the Effective Salience of a Stimulus by Direct and Associative Activation of Its Representation
}

\author{
Geoffrey Hall \\ University of York
}

\author{
José Prados and Joan Sansa \\ University of Barcelona
}

\begin{abstract}
In 2 experiments, rats received exposure to presentations of a footshock preceded by a given cue. In the PRf (partial reinforcement) condition, this cue also occurred in the absence of the shock; in the CRf (continuous reinforcement) condition, it did not. Subsequent testing in which a new stimulus was used to signal the shock (Experiment 1) showed that the shock was more effective as a reinforcer for the PRf than for the CRf group. In Experiment 2, the shock was used as a conditioned stimulus signaling food delivery, and it was found that conditioning occurred more readily in the PRf than in the CRf group. These results accord with the hypothesis that preexposure to the shock results in a decline in its effective salience but that experience of a cue that signals shock in the absence of the shock itself attenuates this effect and helps maintain stimulus salience.
\end{abstract}

Keywords: rats, conditioning, habituation, stimulus salience

Standard theories of classical conditioning assume a conceptual nervous system consisting of a set of representational nodes connected by associative links. These nodes can be activated by direct application of the relevant stimulus and also by way of excitatory associative links when these have been established by prior training. Apart from certain well-defined exceptions, it is assumed that the degree to which presentation of a stimulus induces activity in its node will be directly determined by the salience (which can be equated, in most cases, with the physical intensity) of the stimulus. (The exceptions include accounts of inhibitory learning that suppose that an inhibitory link can raise the threshold for activation of a node; also, Wagner's, 1981, suggestion that a node that is already activated associatively will be less susceptible to activation by an external stimulus.) Recently, however, Hall (2003) has challenged the assumption that salience is a fixed stimulus property, arguing that the effective salience of a stimulus of given intensity can be modified by experience. He suggested that mere exposure to a stimulus will bring about a reduction in its effective salience - that repeated direct activation of a node will reduce the sensitivity of that node. He also suggested that this process could be reversed by presentations of a cue associated with the stimulus - that associative activation of a node (in the absence of the stimulus itself) will act to restore the sensitivity of the node. Put informally, this latter proposal amounts to the suggestion that when an animal has had

Geoffrey Hall, Department of Psychology, University of York, York, United Kingdom; José Prados and Joan Sansa, Department of Basic Psychology, University of Barcelona, Barcelona, Spain

José Prados is now at the School of Psychology, University of Leicester, Leicester, United Kingdom.

This work was supported by a grant from the United Kingdom Biotechnology and Biological Science Research Council. We thank Stuart Morley for technical assistance.

Correspondence concerning this article should be addressed to Geoffrey Hall, Department of Psychology, University of York, York YO10 5DD, United Kingdom. E-mail: g.hall@ @sych.york.ac.uk the experience of expecting an event that does not occur, its sensitivity to a subsequent presentation of that event is enhanced.

The empirical basis for the suggestion that stimulus salience can be modified in this way comes largely from work on the perceptual learning effect (Blair \& Hall, 2003a, 2003b; Mondragón \& Hall, 2002) - from studies of the effects of preexposure to events used as conditioned stimuli (CSs) in discrimination learning procedures. But, as Hall (2003) has noted, there is no reason to suppose that the effects of interest will be restricted to stimuli of this type. The events typically used as unconditioned stimuli (USs) in conditioning experiments differ from those used as CSs in that they are more likely to be of motivational significance and to evoke an overt unconditioned response (UR), but these properties do not preclude the operation of the learning processes that might act to modify stimulus salience. Indeed, in the experiments we report here, we attempted to make use of these properties to investigate Hall's (2003) hypothesis about the conditions under which effective salience is modified.

In both experiments the subjects were rats, and the stimulus of interest was an electric footshock. In each, the animals experienced a series of shock presentations under one of two conditions. In one condition, continuous reinforcement (CRf), each shock was preceded by a conditioned stimulus (CS; a noise or a light). This procedure allows the $\mathrm{CS}$ to acquire associative strength as a signal for shock and should also, according to the hypothesis being investigated, result in a loss of salience by the shock. The CS was included in this condition to allow comparison with the second condition, partial reinforcement (PRf). Animals in this latter condition received the same $\mathrm{CS}$-shock trials as those in the CRf condition, but in addition they received an equal number of nonreinforced trials on which the CS occurred alone. These trials were presented in alternation with the reinforced trials. For these animals, too, the shock is predicted to lose salience each time it is presented (on the reinforced trials), but this loss should be reversed on the trials in which the CS is presented alone. On each of these trials, the node representing the shock is activated associatively (by 
virtue of the excitatory CS-shock association formed on the preceding trial) in the absence of the shock itself, the circumstance hypothesized to enhance the sensitivity of the node. It follows, then, that the salience of the shock should be greater in the PRf condition than in the CRf condition.

In each experiment a different procedure was used to look for a difference between the PRf and CRf conditions in the effective salience of the shock. In Experiment 1, we assessed the ability of the shock to serve as a US by giving both groups a further stage of training in which a novel CS signaled the occurrence of the shock. If salience is lost in the CRf condition but maintained in the PRf condition, acquisition of the conditioned response (CR) should occur more readily in the latter group than in the former. In Experiment 2, we assessed the ability of the shock to serve as a CS by using it to signal the delivery of food. We anticipated that rats that had had previous experience of the shock on the PRf condition would acquire an appetitive CR to this event more readily than those given preexposure to the shock in the CRf condition.

\section{Experiments $1 \mathrm{~A}, 1 \mathrm{~B}$, and $1 \mathrm{C}$}

It is well established that preexposure to a shock will lead to a retardation of subsequent conditioning in which the shock is used as the US (a phenomenon referred to as the US-preexposure effect). In their review of this effect, Randich and LoLordo (1979) discussed two possible mechanisms, one associative and one nonassociative. The associative mechanism depends on the principle of blocking. During the US-preexposure phase, it is likely that an association will be formed between the cues that define the context in which preexposure is given and the shock. As a consequence, the shock US of the conditioning phase will already be predicted by the context, something that might act to restrict the acquisition of associative strength by the experimenter's CS, which is introduced in the test phase of this procedure. Evidence consistent with this suggestion comes from the observation (e.g., Baker, Mercier, Gabel, \& Baker, 1981; Randich, 1981) that the size of the USpreexposure effect can be attenuated when presentations of the shock during the preexposure phase are preceded by a signal. According to standard cue-competition theories of associative learning (e.g., Rescorla \& Wagner, 1972), such a signal will limit the ability of the context to acquire associative strength and should thus reduce the ability of the context to block acquisition by the target CS in the second phase.

The nonassociative mechanism proposed by Randich and LoLordo (1979) is of special interest for our present concerns; it was that mere exposure to the shock might produce habituation, reducing the effective intensity of the shock and thus its power to act as a reinforcer. According to this interpretation, therefore, the US-preexposure effect may be taken as evidence in favor of one aspect of Hall's (2003) analysis of learned changes in stimulus salience. This habituation mechanism can be expected to operate even when the shock is preceded by a signal in the preexposure phase and might thus be expected to make a major contribution to the US-preexposure effect for the CRf group in the present experiment. The question of interest was whether the effect would be attenuated in animals given the PRf condition during preexposure. The hypothesis under investigation predicts that associative activation of the shock node (occurring when the signal is presented without the shock) will reverse the effects of the habituation process that operates on those trials on which the shock itself is presented. The effective salience of the shock should thus be greater in the PRf group than in the CRf group, and conditioning in which this shock serves as the US should proceed more readily in the former group. In Experiment 1A, two groups of rats received preexposure to a shock, one in the PRf condition and one in the CRf condition. We then monitored the acquisition of conditioned suppression over a series of trials in which a different CS (e.g., a light rather than a noise) signaled the shock. We hoped to observe more rapid acquisition by the PRf group. Note that the test procedure used in this experiment involves a change of schedule for the PRf group, a factor that might, in itself, generate any effect observed. Accordingly, in Experiment 1B we repeated the preexposure procedure for the PRf and CRf groups but assessed its effects in a test stage that used a partially reinforced schedule for all the animals. Our hypothesis predicted superior acquisition by the PRf group in this case too. Experiment 1C constituted a replication of Experiment $1 \mathrm{~A}$ but included two further control conditions - a group given equivalent preexposure to unsignaled shocks, and a group given no shock preexposure. We predicted that the latter two groups would allow a demonstration of the standard US-preexposure effect and thus provide a baseline against which the effects of the PRf and CRf treatments could be assessed.

\section{Method}

Subjects. The subjects in Experiment 1A were 16 male hooded Lister rats (Rattus norvegicus) with a mean ad-lib body weight of $320 \mathrm{~g}$ at the start of the experiment. A further 16 naive rats from the same stock (mean ad-lib weight: $505 \mathrm{~g}$ ) were used in Experiment 1B. The subjects in Experiment $1 \mathrm{C}$ were 24 rats from the same stock. Sixteen were experimentally naive and had a mean ad-lib weight of $390 \mathrm{~g} ; 8$ (mean ad-lib weight: $540 \mathrm{~g}$ ) had previously served in a study of flavor aversion learning. Each experimental condition had 4 naive and 2 experienced rats. The rats were housed in pairs in a colony room that was lit from 8:00 a.m. to 8:00 p.m. daily. Experimental treatments were given during the light phase of the daily cycle. Before the start of training, access to food was restricted so that the rats were reduced to and maintained at $85 \%$ of their ad-lib weights.

Apparatus. The apparatus consisted of four operant chambers, supplied by Med Associates (St. Albans, VT). These measured $30 \mathrm{~cm} \times 24$ $\mathrm{cm} \times 21 \mathrm{~cm}$ and were housed in sound-attenuating chests. The ceiling and two longest sides of the chamber were made of clear plastic, and the front and back walls of stainless steel. The front wall of the chamber contained two retractable response levers, $11.5 \mathrm{~cm}$ apart, center to center. The lever on the left was withdrawn from the chamber throughout the experiment. Midway between the levers was an aperture, $5 \mathrm{~cm} \times 5 \mathrm{~cm}$, that gave access to a food cup to which $45 \mathrm{mg}$ of Noyes food pellets could be delivered. Set in the wall above each lever was a $100-\mathrm{mA} 28-\mathrm{V}$ stimulus lamp; simultaneous operation of both lamps constituted the light stimulus (see Procedure section). A shielded houselight (50 mA, $28 \mathrm{~V})$ set high on the rear wall of the chamber provided background illumination. A speaker adjacent to the houselight was used to present white noise at $85 \mathrm{dBA}$ (measured close to the lever). The floor of the chamber consisted of stainless steel rods to which a scrambled shock could be delivered from a Coulbourn Instruments (Allentown, PA) shock source.

Procedure. Training consisted of daily 40-min sessions. In the first five sessions, a lever-press baseline was established. For the first two sessions, each lever press yielded a single food pellet, and in addition, "free" food was delivered according to a variable time (VT) 30-s schedule in the first session and a VT 60-s schedule in the second. Responding was reinforced according to a variable interval (VI) 30-s schedule in Session 3 and a VI 60-s schedule in Sessions 4 and 5. The VI 60-s schedule remained 
in force throughout the rest of the experiment. To attenuate any unconditioned suppression they might produce, we presented, nonreinforced, the events subsequently to be used as CSs in Session 5. There were two stimulus presentations, each 60-s long, the first after $13 \mathrm{~min}$ and the second after $26 \mathrm{~min}$. For half the rats the first stimulus was the noise presentation and the second the light; for the remainder the order was reversed.

There were five sessions of Phase 1 training. The same procedure was used in Experiments 1A and 1B. In each experiment the rats were divided into two equal-sized groups. For the CRf group, each session contained two presentations of a shock ( $0.35 \mathrm{~mA}, 0.5$-s long), each immediately preceded by a 60 -s presentation of Stimulus A. Stimulus presentations occurred 13 min and 26 min after the start of the session. The PRf group received four presentations of Stimulus A per session, the first after $7 \mathrm{~min}$, and at 7-min intervals thereafter. The first and third of these trials were followed by the shock; the second and fourth were nonreinforced. In Experiment 1C, the rats were divided into four equal-sized groups. The CRf and PRf groups received the treatment just described. The shock-only group received two shocks per session, scheduled as for the CRf group. The VI group received further sessions of VI 60-s baseline training.

In Phase 2, all subjects received conditioned suppression training with a different CS, Stimulus B. There were four sessions of training in Experiment 1A and six sessions in Experiment 1C; in both experiments, trials were presented according to the schedule used for the CRf group in Phase 1. To ensure that baseline responding was not disrupted unduly, we reduced the shock intensity to $0.25 \mathrm{~mA}$ on the first of these sessions in Experiment 1A, but because responding continued reliably, we reverted to $0.35 \mathrm{~mA}$ on subsequent sessions and maintained this intensity throughout Experiments 1B and 1C. In Experiment 1B, trials were presented according to the schedule used for the PRf group in Phase 1. Conditioning proceeded less rapidly with this schedule, and hence, 8 sessions were given. In Experiment $1 \mathrm{~A}$, half the rats in each group received the noise as Stimulus A and the light as Stimulus B, whereas for the remainder this arrangement was reversed. Because the results of this experiment revealed no obvious difference between these counterbalanced subgroups, we used the noise as Stimulus A and the light as Stimulus B for all subjects in Experiments 1B and 1C. Responding was recorded separately during each CS and the 60-s stimulus-free period that preceded each CS (the pre-CS period). Responses recorded on the relevant trials of each session were pooled, allowing the computation of a daily suppression ratio of the form $a / a+b$, where $a$ represents responses occurring during the CS presentations, and $b$ represents responses occurring in the pre-CS periods.

\section{Results and Discussion}

Experiment 1A. Phase 1 training in Experiment 1A resulted in the acquisition of suppression to Stimulus A in both groups. Perhaps surprisingly, given the different reinforcement schedules they experienced, there was little difference between the two groups. The suppression ratios recorded on the reinforced trials of Sessions $1-5$ were $.38, .42, .35, .33$, and .20 for the PRf group and $.44, .45, .38, .33$, and .20 for the CRf group. An analysis of variance (ANOVA) with group and session as the variables revealed only a significant effect of session, $F(4,56)=9.49$ (other $F_{\mathrm{s}}<1$ ). (Here and elsewhere, a significance level of $p<.05$ was adopted.) Baseline response rates were also closely similar; the rates recorded over all the pre-CS periods that preceded reinforced trials in Phase 1 were 15.21 responses per minute for the PRf group and 17.01 responses per minute for the CRf group. These scores did not differ significantly $(F<1)$.

The differing Phase 1 treatments produced a marked difference in Phase 2. Figure 1 shows group mean suppression ratios for the four sessions of this phase. It is evident that the PRf group acquired suppression to Stimulus B more readily than did the CRf group. An

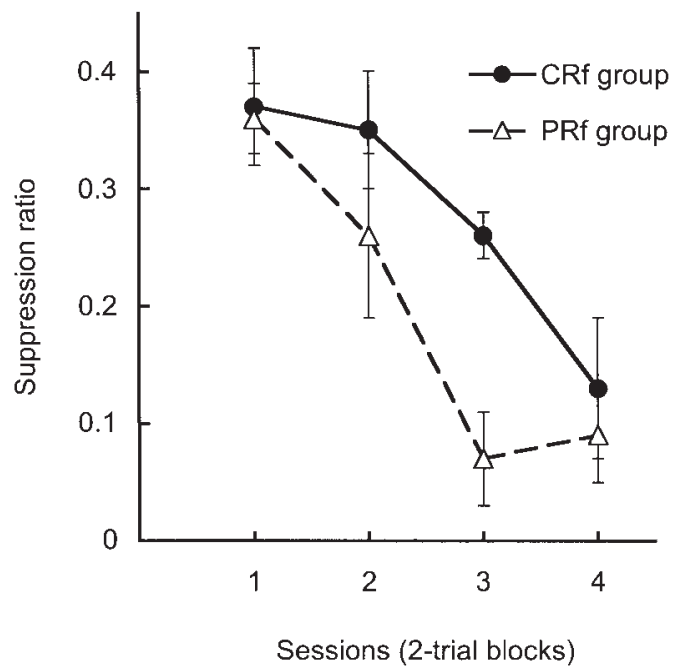

Figure 1. Experiment 1A: Group mean suppression ratios for Phase 2. Each session contained two presentations of Conditioned Stimulus B, each followed by shock. The continuous reinforcement (CRf) group had been preexposed in Phase 1 to shock presentations preceded by Conditioned Stimulus A; the partial reinforcement (PRf) group received both A-shock trials and also nonreinforced presentations of A in Phase 1. Vertical bars represent standard errors of the means.

ANOVA conducted on the scores summarized in Figure 1 showed significant effects of session, $F(3,42)=13.65$, and of group, $F(1$, $14)=4.66$. The interaction between the variables was not significant, $F(3,42)=1.59$. Baseline responding was maintained at the same level for the two groups. The mean rates, pooling over all pre-CS periods in Phase 2, were 21.21 responses per minute in the PRf group and 20.63 responses per minute in the CRf group $(F<1)$.

The result depicted in Figure 1 is consistent with the proposal that the effective salience of the shock is higher, as a result of Phase 1 training, in the PRf group than in the CRf group, but we must consider possible alternative explanations. One possibility is that the effect obtained might be mediated by differences between the groups in the associative strength of the context. If the CRf procedure leaves the context with more strength than does the PRf procedure, then the relatively poor acquisition to Stimulus B shown by the CRf group might be interpreted as a consequence of blocking by context (the mechanism proposed as one possible source of the basic US-preexposure effect). There are two reasons to doubt this interpretation. First, a straightforward application of standard cue-competition theory (e.g., Rescorla \& Wagner, 1972) to our Phase 1 training procedure leads to the conclusion that the PRf procedure (in which Stimulus A is a rather poor predictor of the occurrence of the shock) would result in the context acquiring greater strength than would the CRf procedure. Second, any sizable difference in the strength of the context might be expected to influence behavior in the presence of the contextual cues, producing a lower level of baseline responding in the more fear-evoking context. But, as we have seen, baseline response rates were closely similar in the two groups in this experiment. Experiment $1 \mathrm{C}$ provides further information relevant to this matter.

Experiment $1 B$. A second possibility is that the shock was equally effective in both groups during Phase 2, but that the change 
of conditions (from the PRf to the CRf schedule) had the effect of promoting the acquisition of suppression to Stimulus B in the PRf group. Experiment 1B was designed to address this issue.

As in Experiment 1A, the PRf and CRf groups differed little in the suppression they acquired to Stimulus A during Phase 1. Mean suppression ratios recorded over the two reinforced trials on each of Sessions 1-5 were .32, .25, .19, .15, and .13 for the PRf group and $.38, .27, .24, .15$, and .07 for the CRf group. An ANOVA with group and session as the variables showed only a significant effect of session, $F(4,56)=12.49$ (other $F \mathrm{~s}<1$ ). The baseline response rate for the PRf group (recorded during the pre-CS periods prior to the reinforced trials of Phase 1) was 14.84 responses per minute; the equivalent rate for the CRf group was 19.95. These scores did not differ reliably, $F(1,14)=2.47$.

Figure 2 shows group mean daily suppression ratios for the second phase of training, responses being pooled over all trials on each session prior to calculation of the ratio (ratios derived from just the reinforced trials on each session generated the same overall picture). It is evident that, as in Experiment 1A, suppression in Phase 2 was more profound in the PRf group than in the CRf group. An ANOVA conducted on the data summarized in the figure showed significant effects of session, $F(7,98)=19.46$, and of group, $F(1,14)=7.23$; the interaction was not significant, $F(7$, $98)=1.31$. The groups did not differ in their baseline response rates. The mean rates, over all pre-CS periods in this phase, were 16.55 responses per minute in the PRf group and 15.50 responses per minute in the CRf group $(F<1)$. We conclude that the shift from one schedule to another is not responsible for the effects seen in Phase 2 of these experiments; what matters, rather, is the nature of the schedule experienced in Phase 1.

Experiment 1C. An error on the part of the experimenter meant that data were not recorded for the first four sessions of

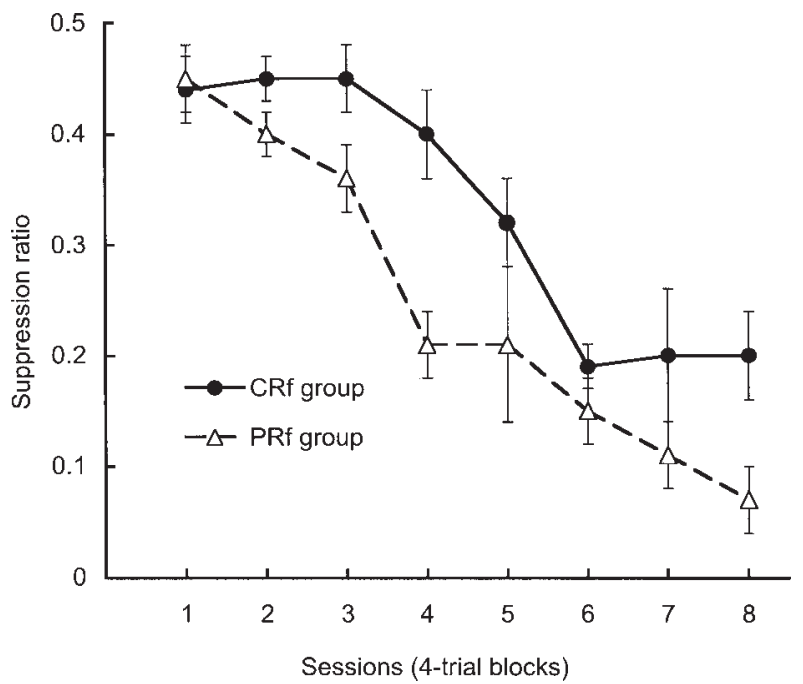

Figure 2. Experiment 1B: Group mean suppression ratios for Phase 2. Each session contained four presentations of Conditioned Stimulus B, two followed by shock and two nonreinforced. The continuous reinforcement (CRf) group had been preexposed in Phase 1 to shock presentations preceded by Conditioned Stimulus A; the partial reinforcement (PRf) group received both $\mathrm{A}$-shock trials and also nonreinforced presentations of $\mathrm{A}$ in Phase 1. Vertical bars represent standard errors of the means.
Phase 1. The mean response rates on the last session of this phase (recorded in pre-CS periods for the CRf and PRf groups, in the equivalent periods for the shock-only group, and across the entire session for the VI group) were 12.50 responses per minute for the PRf group, 12.08 responses per minute for the CRf group, 11.75 responses per minute for the shock-only group, and 10.19 responses per minute for the VI group. These scores did not differ reliably $(F<1)$. Training with $\mathrm{CS}$ A established suppression to this stimulus, which, in this experiment, was somewhat more profound in the CRf group (with a mean suppression ratio of .16 on the last session of Phase 1) than in the PRf group (which had a mean of .25 on this session). The difference between these means was not, however, statistically significant $(F<1)$.

The results for the acquisition of conditioned suppression to Stimulus B in Phase 2 are shown in Figure 3. As in the previous experiment, the PRf group acquired suppression more readily than the CRf group. The VI group (for whom the US was novel at the start of Phase 2) acquired suppression more readily than any of the other groups. The difference between this group and the shockonly group constitutes an example of the standard US-preexposure effect. There was no sign that signaling the US during preexposure attenuated the size of the US-preeexposure effect-the PRf and shock-only groups learned at much the same rate, and acquisition was slowest of all in the CRf group.

An ANOVA conducted on the data summarized in Figure 3 revealed significant main effects of group, $F(3,20)=4.14$, of session, $F(5,20)=40.95$, and of the interaction between these variables, $F(15,100)=2.45$. Analysis of simple main effects showed a difference among the groups on Sessions 2, 3, 4, and 5: smallest $F(3,120)=3.85$. There were no differences on Sessions 1 and $6(F \mathrm{~s}<1)$. Pairwise comparisons using Tukey's test showed the following differences to be significant: between the VI group and the PRf and CRf groups on Session 2, the CRf and VI groups on Session 3, the CRf group and each of the other groups on Session 4, and the CRf group and the VI and shock-only groups on Session 5.

These results successfully replicated those of Experiment 1A, in that the CRf group learned less readily than the PRf group. They also demonstrated the standard US-preexposure effect, with the shock-only group learning less readily than the VI group. What was unexpected, however, was that we failed to replicate the effects of signaling the shock in the exposure phase, previously reported by others (Baker et al., 1981; Randich, 1981). The CRf group, which received a preexposure treatment similar to that used in earlier studies, not only failed to show an attenuation of the US-preexposure effect but also learned significantly more slowly than the other groups. We can only speculate as to why this should have occurred. One possibility arises from a consideration of the role of shock magnitude. Previous researchers have, for the most part, used more severe shocks than those used in our experiments. Baker et al. (1981) used shocks of 1-mA and 0.5-s duration or of $0.5 \mathrm{~mA}$ with a duration of $1.3 \mathrm{~s}$; Randich used shocks of $0.8 \mathrm{~mA}$ and $0.8 \mathrm{~s}$ or of $1 \mathrm{~mA}$ for $0.8 \mathrm{~s}$ (our shocks were of $0.35 \mathrm{~mA}$ for $0.5 \mathrm{~s}$ ). When he used only a weak shock in preexposure, Randich (1981, Experiment 4) found that signaling it did not attenuate the US-preexposure effect. He suggested in explanation that only stronger shocks will support a substantial amount of context conditioning and that only with these is the US-preexposure effect primarily a consequence of blocking by contextual cues (and thus 


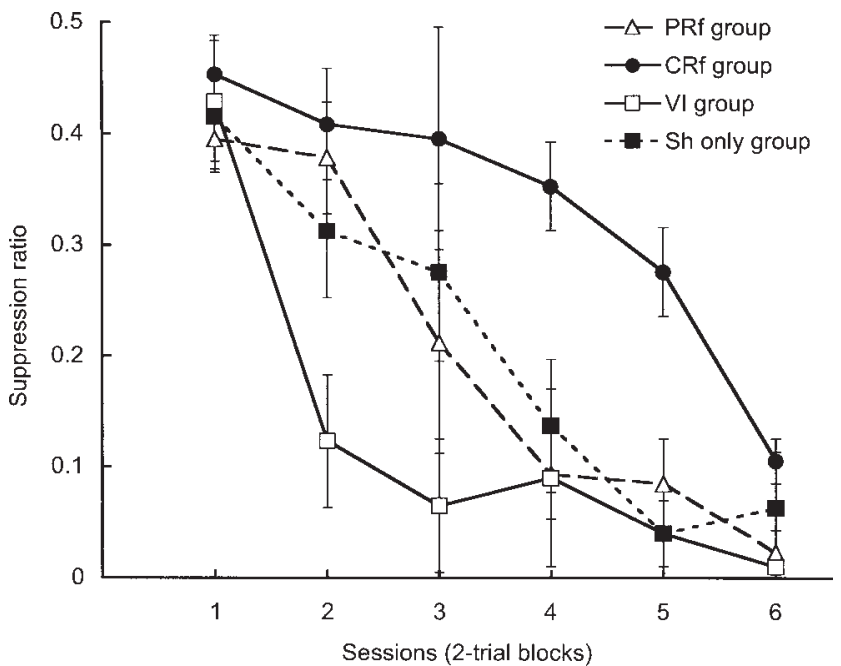

Figure 3. Experiment 1C: Group mean suppression ratios for Phase 2. Each session contained two presentations of Conditioned Stimulus B, each followed by shock. The continuous reinforcement (CRf) group had been preexposed in Phase 1 to shock presentations preceded by Conditioned Stimulus A; the partial reinforcement (PRf) group received both A-shock trials and also nonreinforced presentations of A in Phase 1. The shock-only (Sh only) group experienced just the shock. The variable interval (VI) group experienced neither the conditioned stimulus nor the shock in Phase 1. Vertical bars represent standard errors of the means.

susceptible to modification by the presence of a signal). A weaker shock, he suggested, will habituate more readily, precluding substantial context conditioning; in this case the US-preexposure effect is taken to be a consequence of the habituation itself. Accepting this analysis lends support to the conclusion that the effects seen in our experiments are likely to be primarily a consequence of habituation to the shock.

What remains unexplained is why there should be no difference between the PRf and shock-only groups in the test phase, with both learning more readily than the CRf group. One possibility emerges from the salience modulation hypothesis that motivated these experiments. Although our analysis of baseline response rates has provided no evidence for differences among the groups in context conditioning, it is theoretically plausible that such conditioning would be stronger in the shock-only group than in the groups for which the shock was signaled (perhaps being quite absent in the CRf group). If so, then associative activation of the shock representation might be expected to occur during Phase 1 training in both the shock-only and the PRf groups. In the latter case, this would occur primarily on nonreinforced presentations of CSA; in the former case, during the intertrial intervals spent in the presence of the conditioned contextual cues. For both groups, then, according to the hypothesis, US habituation would be attenuated. For the CRf group, lacking context-US associations and receiving no nonreinforced presentations of CSA, habituation would proceed unhindered.

Whatever the merits of this speculation, it is appropriate to consider alternative possible interpretations of the pattern of results generated by Experiment 1C. One possibility can be derived from Wagner's (1981) theory, provided it is allowed that there might be substantial generalization between the events used as CSs in the two phases of the procedure. According to this theory, a well-trained CS will "prime" the representation of the US into a secondary state of activation (the A2 state), making presentation of that US less effective as a reinforcer for association formation. A CS trained in the CRf condition might be expected to be a more effective prime than one trained according to the PRf schedule and, accordingly, generalization from Stimulus A (trained in Phase 1) to Stimulus B (the CS used in Phase 2) will mean that the US will be less effective, and Phase 2 conditioning will proceed less readily, in the CRf than in the PRf group. It is perhaps a problem for this analysis that in none of our experiments was there a clear difference between the PRf and CRf groups in the acquisition of suppression to Stimulus A in Phase 1 of training.

Another possibility, which also depends on the assumption that there will be generalization between Stimuli A and B, can be derived from consideration of the role of latent inhibition. The CRf and PRf groups of this experiment differed from the other two groups in that they received repeated presentations of a CS (a noise) in Phase 1. If latent inhibition accrued to the noise CS in Phase 1 and generalized to the light CS used in Phase 2, then an additional factor would be operating to retard Phase 2 acquisition in the PRf and CRf groups. That the retardation should be more profound in the CRf group than in the PRf group (in spite of the fact that the latter group received more CS presentations and thus potentially accrued more latent inhibition) is not necessarily a problem. According to Pearce and Hall (1980; see also Hall \& Pearce, 1982; Pearce, Kaye, \& Hall, 1982), the development of latent inhibition to a stimulus will be modified by events that follow stimulus presentations. In particular, Pearce and Hall have suggested that a stimulus that is followed by inconsistent consequences will suffer less latent inhibition than one that is followed by consistent consequences. What follows is that Stimulus A should acquire more latent inhibition in the CRf group than in the PRf group. If there were substantial generalization between Stimulus A and Stimulus B, this might be enough to explain why the CRf group should condition more readily than the PRf group in Phase 2-the difference between them would be a consequence of a difference in the properties of the CS rather than in the US, as we have been supposing. The experiments to be reported next provide data that bear on this issue.

\section{Experiments 2A, 2B, and 2C}

Although shock is usually used as a US in conditioning experiments, in principle there is no reason why it should be unable to serve as a CS predicting the occurrence of some other event. In unpublished experiments, we have investigated a counterconditioning procedure in which the occurrence of a shock was followed, after $10 \mathrm{~s}$, by the delivery of a food pellet; we monitored, as the CR, the rat's tendency to approach the food tray in the postshock interval. Footshock evokes a variety of URs in the rat. The most obvious is the so-called postshock activity burst (Fanselow, 1982), in which the rat shows head movement and turns about its vertical axis but is otherwise immobile. These responses would preclude approaching the food tray, and our studies showed that, for our training procedure, the first presentation of a shock produced a suppression of responding that lasted at least $10 \mathrm{~s}$. With repeated shock presentations, however, this 
post-US suppression disappeared and was replaced by an increasing tendency to approach the food tray in the postshock interval.

In Experiment 2A, we report the results of a study using this procedure, in which two different shock intensities were compared. It showed, to anticipate, that the stronger shock evoked slightly more suppression of behavior on initial presentation but that, thereafter (as might be expected of a more salient CS), it supported more rapid acquisition of the appetitive CR. The choice of shock intensities for use in this experiment was guided by the hypothesis that pretraining with the $0.35-\mathrm{mA}$ shock (as was given in Experiment 1) reduces the effective intensity of the shock, and it does so more substantially in the CRf than in the PRf condition. Accordingly, we used shock intensities of $0.3 \mathrm{~mA}$ and $0.2 \mathrm{~mA}$, the former intended to mimic the CRf condition of the previous experiments, and the latter the PRf condition.

In Experiment 2B, we used this procedure to assess the effects of the PRf and CRf preexposure schedules on the effectiveness of the $0.35-\mathrm{mA}$ shock as a CS. If preexposure in the PRf condition leaves the shock more salient than does preexposure in the CRf condition, then it is possible that the development of the appetitive $\mathrm{CR}$ of approaching the food tray will proceed more rapidly in the PRf than in the CRf group.

Experiment $2 \mathrm{C}$ constituted an attempt to replicate the essential features of the results of the PRf and CRf groups of Experiment 2B. In addition, two further conditions were investigated. These received the PRf and CRf treatments in the first phase of training, but in the test phase the event used as the CS was the brief (0.5-s) presentation of a neutral stimulus (noise or light). (This duration was chosen to match that of the shock CS in the other groups.) If the effects of the CRf and PRf treatments are indeed the consequence of changes in the effective salience of the shock, they should be without effect when some event other than the shock is used as the CS in the test phase.

\section{Method}

Subjects and apparatus. The subjects for Experiment 2A were 16 naive male hooded rats with a mean ad-lib weight of $335 \mathrm{~g}$ at the start of the experiment; a further 16 (mean ad-lib weight: $395 \mathrm{~g}$ ) were used in Experiment 2B, and a further 32 (mean ad-lib weight: $505 \mathrm{~g}$ ) were used in Experiment 2C. The apparatus was the same as that used in Experiment 1, except that no response lever was available. An infrared beam and photocell were used to record entries into the food tray.

Procedure. At the start of Experiment 2A, all subjects received a single 40-min session of magazine training in which food pellets were delivered according to a VT 60 -s schedule. They then received five sessions of training in which shock signaled food. There were two shocks per session, occurring after $13 \mathrm{~min}$ and $26 \mathrm{~min}$, each of which was followed, after an interval of $10 \mathrm{~s}$, by the delivery of a food pellet. For half the rats the shock intensity was $0.3 \mathrm{~mA}$, and for half it was $0.2 \mathrm{~mA}$. Responses to the food tray were recorded separately for the 10-s period preceding food delivery (the CS interval) and for the 10-s period (the pre-CS period) preceding the shock. In details not specified here, the procedure was the same as described for the previous experiments.

In Experiment 2B, we used the conditioning procedure just described, except that shock intensity for all subjects was $0.35 \mathrm{~mA}$ (the intensity used in Experiment 1). Prior to this test, all subjects received five sessions of preexposure to the shock. These sessions were organized as described for Experiment 1A, apart from the absence of the baseline lever-press response. Thus, half the animals received the PRf schedule and half the CRf schedule; for half of each of these groups the CS (Stimulus A) was the light, and for half it was the noise.
The preexposure procedure used in Experiment $2 \mathrm{C}$ was identical to that just described, with 16 rats receiving the CRf schedule and 16 the PRf schedule. For the next phase of training (eight sessions) the animals were divided into four groups. Two received training in which, as before, the shock signaled food. One of these groups (the PRf-shock group) had received the PRf procedure in the first phase, and the other (the CRf-shock group) had received the CRf treatment. Half the animals in each of these groups had experienced the light in Phase 1, and half had experienced the noise. The remaining two groups (the PRf-B and CRf-B groups) received similar training except that the food pellet was signaled not by shock but by a 0.5 -s presentation of Stimulus B (which was the noise for those that had experienced the light as CS A in the first phase and the light for those that received the noise as $\mathrm{CS} \mathrm{A}$ )

\section{Results and Discussion}

Experiment 2A. Figure 4 shows group mean response rates during the CS interval for the five sessions (two-trial blocks) of training. The score used on each block was corrected for any differences in baseline rates of response by subtracting responses made in the corresponding pre-CS periods. In fact, responding outside the CS period was relatively infrequent. The mean pre-CS response rates over all sessions were 2.85 responses per minute for the $0.3-\mathrm{mA}$ group and 2.93 responses per minute for the $0.2-\mathrm{mA}$ group. These rates did not differ significantly $(F<1)$.

As the figure shows, postshock responding tended to be suppressed on the first session, but thereafter, responding increased in both groups over the course of training. The initial suppression was somewhat more marked for the group given the stronger shock, but this group then showed substantially more rapid acquisition of the magazine entry response. An ANOVA with group and session as the variables showed no significant effect of group, $F(1,14)=$ 1.52 , but there was a significant effect of session, $F(4,14)=$ 10.01 , and a significant interaction between the variables, $F(4$, $56)=3.11$. Analysis of simple main effects showed that the groups differed significantly on the final session, $F(1,70)=4.75$; the difference apparent on Session 1 was not statistically reliable, $F(1,70)=2.42$. These results confirmed that appetitive condi-

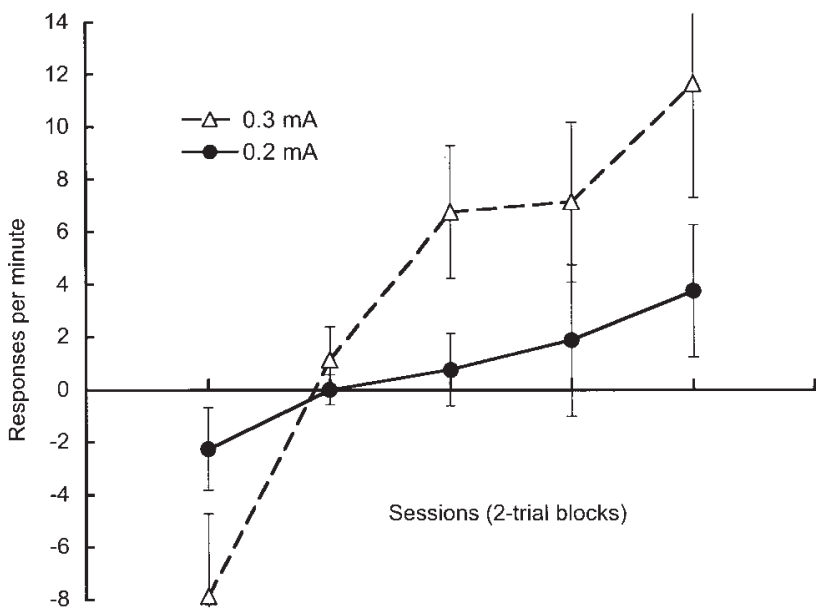

Figure 4. Experiment 2A: Group mean response rates during the 10-s prefood periods. The conditioned stimulus was the presentation of a shock of either $0.3 \mathrm{~mA}$ or $0.2 \mathrm{~mA}$. Vertical bars represent standard errors of the means. 
tioning can be obtained with the present training procedure and that, for the shock levels used here, learning occurred more rapidly with the stronger shock. This finding is, of course, specific to the particular shock levels used in this experiment-with a much stronger shock it is quite possible that the postshock suppression would be profound enough to outweigh any advantage produced by its greater salience as a CS, and in these circumstances an appetitive CR might develop only slowly. It should be noted, however, that the shock levels used here were chosen to be within the range that is of interest on the basis of the results of Experiment 1.

Experiment $2 B$. No data were recorded during the first phase of training. Figure 5 shows the acquisition of responding to the food tray in the second phase of training. The pattern of results is strikingly similar to that observed in Experiment 2A, with the PRf group (like the 0.3-mA group of Experiment 2A) showing some initial suppression followed by rapid development of responding, and the CRf group (like the 0.2-mA group of the previous experiment) showing less initial suppression and slower subsequent acquisition. An ANOVA revealed no significant main effect of group, $F(1,14)=2.07$, but a significant effect of session, $F(4$, $14)=27.09$, and a significant interaction between the variables, $F(4,56)=9.04$. Analysis of simple main effects showed that the groups differed significantly only on Session $4, F(1,70)=5.60$. Again the difference between the groups on Session 1 fell short of significance, $F(1,70)=2.95, p<.10$. Background response rates recorded during pre-CS periods were 1.73 responses per minute in the PRf group and 0.90 responses per minute in the CRf group. These scores did not differ significantly, $F(1,14)=1.45$.

These results show that the shock was more effective as a CS when it had been preexposed according to the PRf as opposed to the CRf schedule. Given the results of Experiment 2A, which show for shock intensities in the range of interest here that a stronger

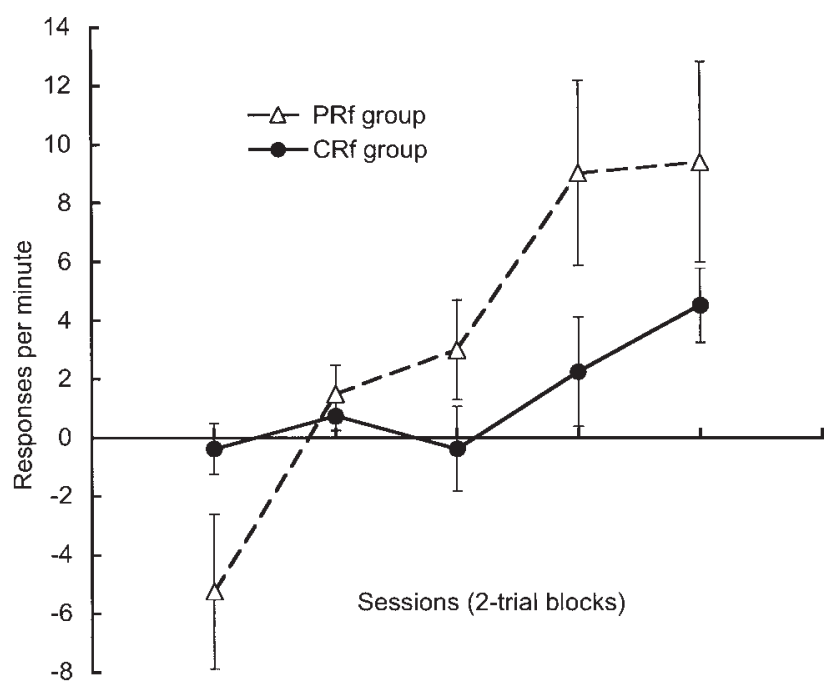

Figure 5. Experiment 2B: Group mean response rates during the 10-s prefood periods in Phase 2. The continuous reinforcement (CRf) group had been preexposed in Phase 1 to shock presentations preceded by Conditioned Stimulus A; the partial reinforcement (PRf) group had received both A-shock trials and also nonreinforced presentations of $\mathrm{A}$ in Phase 1. Vertical bars represent standard errors of the means. shock is a more effective CS than a weaker shock, this outcome is consistent with the hypothesis that the effective salience of the shock is modified by preexposure and is higher after the PRf than after the CRf treatment.

Experiment 2C. Figure 6 shows group mean response rates during the CS interval for the four two-session blocks of the appetitive conditioning phase. The mean pre-CS rates during this phase were 2.44 responses per minute for the PRf-shock group, 1.88 for the CRf-shock group, 3.14 for the PRf-B group, and 2.34 responses per minute for the CRf-B group. These rates did not differ significantly $(F<1)$. There was no indication in this experiment that postshock suppression was initially more profound in the PRf-shock group than in the CRf-shock group; in fact, the reverse appeared to be the case. To maintain comparability with the previous experiments, we analyzed performance on the very first session for CRf-shock and PRf-shock groups. The scores of -3.75 responses per minute for the former and 0.75 responses per minute for the latter did not differ significantly $(F<1)$. Further work that uses a more sensitive measure will be needed to establish the true nature of the effects of preexposure on the URs elicited by shock.

Figure 6 shows that responding in the CS periods increased in all groups over the course of Phase 2. There was no difference between the two groups trained with Stimulus B as the cue. But, as in Experiment 2B, for animals trained with shock as the cue, learning occurred more rapidly in the PRf group than in the CRf group. Statistical analysis confirmed this description. An ANOVA was conducted on the data summarized in the figure, with type of stimulus (shock or B), preexposure procedure (PRf or CRf), and block as the variables. There was a main effect of block, $F(3$, $28)=22.6$, but not of stimulus $(F<1)$ or of preexposure schedule, $F(1,28)=3.34, .05<p<.10$. There was, however, a significant interaction between stimulus type and preexposure procedure, $F(1,28)=5.95$ (for the other interactions, $F \mathrm{~s}<1)$. An analysis of simple main effects confirmed the significance of the difference between the two groups trained with shock as the CS, $F(1,28)=9.10$; there was no difference between the groups trained with Stimulus B as the CS $(F<1)$.

These results confirm those of Experiment $2 \mathrm{~B}$, in showing that the shock was more effective as a CS when it had been preexposed under the PRf as opposed to the CRf schedule. This outcome is not the consequence of some (unspecified) general process by which exposure to the PRf schedule enhances any form of subsequent conditioning - when an event other than the preexposed shock was used as the CS, there was no sign of a difference between the PRf and CRf groups.

This pattern of results also speaks to the possibility, raised in the discussion of Experiment 1, that the effects obtained in that experiment might be a consequence of the generalization of latent inhibition. Recall that the account of associability change proposed by Pearce and Hall (1980) predicts that the level of associability governed by the CS at the end of Phase 1 will be higher in the PRf group than in the CRf group. Generalization from the CS used in Phase 1 to that used in Phase 2 (i.e., from light to noise or vice versa) might thus account for the more rapid learning shown by the PRf group in Experiment 1. This interpretation receives no support, however, from the results of the present experiment. On the face of things, generalization of latent inhibition from Stimulus A to Stimulus B should operate in this experiment too, and it should 


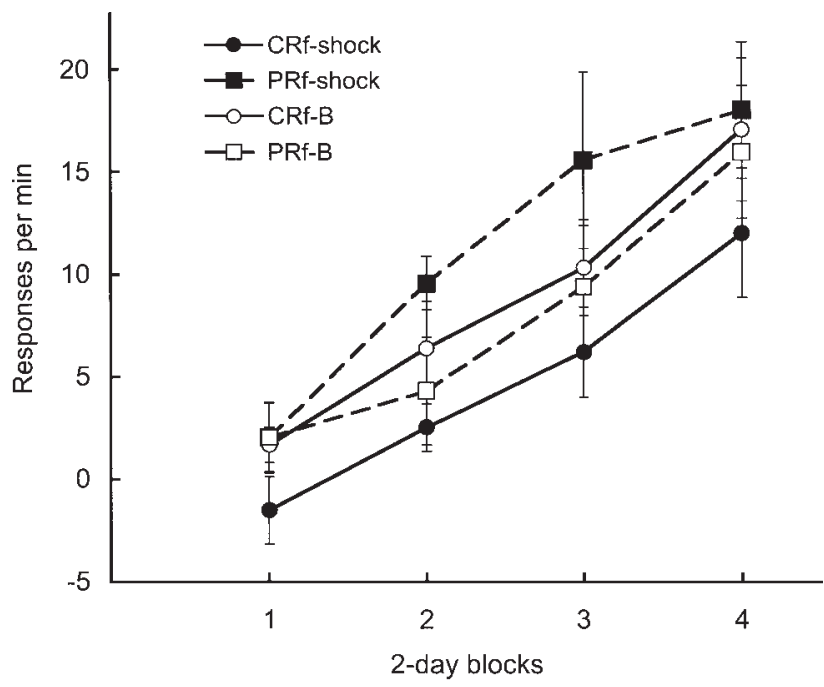

Figure 6. Experiment 2C: Group mean response rates during the 10-s prefood periods in Phase 2. The continuous reinforcement (CRf) group had been preexposed in Phase 1 to shock presentations preceded by Conditioned Stimulus A; the partial reinforcement (PRf) group had received both A-shock trials and also nonreinforced presentations of A in Phase 1. For the shock groups, the conditioned stimulus was the presentation of a shock; for the B groups, it was a 0.5 -s presentation of Stimulus B (noise or light). Vertical bars represent standard errors of the means. $\min =$ minute.

result in a difference in test performance between the PRf-B and CRf-B groups, whereas no such difference was found. And the fact that the result of interest from Experiment 1-better test performance after PRf than after CRf preexposure-was reproduced in this experiment in animals that experienced the shock, rather than light or noise, as the CS, is comfortably accommodated by the proposal that in both experiments, the effects obtained are a consequence of changes in the effectiveness of the shock itself. We acknowledge, however, that the procedure used in this experiment (in which the CS duration was reduced from $60 \mathrm{~s}$ for Stimulus A in Phase 1 to $0.5 \mathrm{~s}$ for Stimulus B in Phase 2) may have acted to obscure generalization between noise and light in the PRf-B and CRf-B groups. If so, generalization between the two stimuli would remain a possibility as an explanation for the results of Experiment 1 , although a different mechanism would be required to explain the difference between the PRf-shock and CRf-shock groups that was found in the present experiment.

\section{General Discussion}

The experiments reported here were designed to investigate the proposal that the effective salience of a stimulus (in this case a shock) could be modified by experience (Hall, 2003). The specific hypothesis being tested was that presentation of a stimulus would produce a reduction in its effective salience, but that activation of the central representation of that stimulus in the absence of the event itself would tend to enhance salience (or attenuate the loss produced by direct experience of the event). Activation of a representation in the absence of the event can be achieved by presenting a signal for that event, and this was the procedure used for the PRf groups of these experiments. These animals received pairings of a CS and the shock, allowing both the formation of a CS-shock association and the possibility of a reduction in shock salience; they also received separate presentations of the CS alone, allowing activation of the shock representation in the absence of shock and thus a possible restoration of salience. For control subjects (the CRf groups), there were no separate presentations of the CS.

The results showed that the shock was a more effective US after PRf than after CRf preexposure (Experiment 1), consistent with the hypothesis that the effective salience of the shock was higher after the PRf than after the CRf treatment. For both groups, subsequent conditioning proceeded less readily than in a group given no preexposure (Experiment 1C), suggesting that the PRf treatment attenuates but does not abolish the loss of effectiveness produced by the CRf treatment. The difference between the PRf and CRf conditions was also evident when the shock was used as a CS in Experiment 2. The more rapid acquisition shown by the PRf group is what would be expected if the effective salience of the shock was higher in this group than in the CRf group.

The interpretation offered for Experiment 1 in terms of the modulation of a loss of salience produced by exposure to the shock fits well with our current understanding of the nature of the US-preexposure effect. It is generally accepted that such preexposure will engage an habituation process that attenuates the impact of the shock (in other words, reduces its effective salience). We acknowledge, however, that the preexposure procedure might result in the formation of a context-shock association that could play a role in determining the outcome of subsequent tests. (Indeed, Wagner, 1981, has suggested that long-term habituation effects may be entirely a product of such associative learning.) The essence of the alternative analysis is that the effectiveness of a shock will be determined by the extent to which it is surprising or unexpected, and that the formation of a context-shock association will make the occurrence of a shock in that context less surprising and, therefore, less effective. It seems unlikely, however, that the effects observed in the experiments reported here could be a consequence of a difference between the PRf and CRf groups in the strength of the context-shock association. The results obtained require the assumption that the shock is more surprising (that the context association is weaker) after the PRf than after the CRf treatment. But as we have already noted, current theories suggest that, if anything, the context-shock association will be weaker in the CRf than in the PRf condition. Furthermore, in none of our experiments was there any indication that the PRf and CRf groups differed in their baseline levels of responding (i.e., in their rates in the presence of the contextual cues).

The extension of the notion of salience modulation to the explanation of the results of Experiment 2 requires further comment. Experiment 2 made use of the latent inhibition paradigm (in that it involved giving preexposure to the event subsequently used as the CS), and the latent inhibition effect has often (but see Wagner, 1981) been explained in terms of a process different from that involved in habituation. Thus, for example, Pearce and Hall (1980) attribute latent inhibition to changes in the value of a CS-associability parameter (alpha), distinct from the salience parameter determined by the physical intensity of the stimulus. It is possible, then, that the effects seen in Experiment 2 are a consequence of changes in the associability parameter and that this parameter is (coincidentally?) sensitive to the same factors (i.e., 
those involved in the PRf and CRf schedules) that also modulate a different process that is responsible for the US-preexposure effect. We cannot refute this hypothesis, but we can point out that it requires the novel assumption that the value of alpha will depend on the way in which the critical CS has been signaled during preexposure. In previous theories of associability change, researchers (e.g., Pearce \& Hall, 1980; see also Mackintosh, 1975) have argued, rather, that associability is determined by the extent to which the CS reliably predicts its consequences. We might also argue that the principle of parsimony favors the attempt to explain the results of both Experiments 1 and 2 in terms of the operation of the same mechanism. We acknowledge, however, that the force of this latter argument is weakened by the fact that this analysis implies that two different processes may be involved in the latent inhibition effect, one that controls changes in associability and another that determines changes in effective salience.

The theoretical view being developed here, that associative activation of a US representation will change the properties of that representation, has something in common with the account of extinction put forward some years ago by Rescorla and his colleagues (e.g., Rescorla \& Cunningham, 1977; Rescorla \& Heth, 1975). Their account, based on studies of the phenomenon known as reinstatement, held that the response decrement produced by nonreinforced presentations of a CS might be a consequence, in part, of a degradation of the representation of the US. Subsequent work, however, has failed to support this interpretation, suggesting instead that the reinstatement effect is a consequence of changes in the strength of the context-US association (see, e.g., Bouton \& King, 1983, 1986). Furthermore, direct tests of Rescorla's hypothesis have usually found that extinction of one CS does not weaken the responding controlled by another CS that has been associated with the same US (Kasprow, Schachtman, Cacheiro, \& Miller, 1984; Richards \& Sargent, 1983) — the outcome that would be expected if the extinction procedure produced a degradation of the US representation. (But see also Kehoe, Weidemann, \& Dartnall, 2004.)

These results have implications for our own theoretical analysis. We have argued that associative activation of a US representation in the absence of a US will reverse the loss of effective salience produced by previous US presentations, making the US more effective when it is presented again. It is an open question as to whether this effect is a consequence of a general change in the sensitivity of the US node. The fact that extinction of one CS does not necessarily modify the CR controlled by another CS associated with that node suggests that it is not-the effect appears to be restricted to cases in which the US itself is presented. This conclusion must be tentative, however, based as it is on a null result (i.e., the failure to find an effect of extinction of one CS on the CR controlled by another). In fact, given that the associative changes generated by extinguishing one CS seem likely to generalize to another, the common failure to find any effect might be taken to indicate the presence of some other process that opposes the effect of generalized extinction.

As was noted in the beginning of this article, the motivation for the experiments reported here arose from a desire to explain effects obtained in experiments on the perceptual learning effect. In studies of this effect, we have found that preexposure in which two similar stimuli are presented in alternation appears to enhance the effective salience of their unique features (e.g., Blair \& Hall, 2003a, 2003b; Mondragón \& Hall, 2002). That is, alternating presentations of stimuli that may be represented as $\mathrm{AX}$ and $\mathrm{BX}$ (where A and B stand for the unique features of these stimuli and $\mathrm{X}$ for those features that, being similar, they hold in common) results in an enhancement of the salience of $\mathrm{A}$ and $\mathrm{B}$ (relative to a control condition in which $\mathrm{AX}$ and $\mathrm{BX}$ are presented on separate blocks of trials). This characterization of the stimuli makes it apparent (see McLaren, Kaye, \& Mackintosh, 1989) that preexposure will allow the formation of the within-compound associations $\mathrm{X}-\mathrm{A}$ and $\mathrm{X}-\mathrm{B}$. The alternating schedule thus ensures that the representation of each unique element will be activated associatively (by virtue of the presence of $\mathrm{X}$ ) on those trials on which it is not actually presented. The PRf schedule of the present experiments constitutes an analog to this situation, with the critical stimulus, in this case the shock, being presented on half the trials, and its representation being activated associatively on intervening trials. The present results show that the effective salience of the shock is maintained by this procedure. If we allow that the same principles will apply when the stimuli in question are not of particular motivational significance, it follows that the intermixed preexposure schedule used in studies of perceptual learning should maintain the effective salience of the unique features of the preexposed stimuli, thus enhancing discrimination between themthe essence of the perceptual learning effect.

\section{References}

Baker, A. G., Mercier, P., Gabel, J., \& Baker, P. A. (1981). Contextual conditioning and the US preexposure effect in conditioned fear. Journal of Experimental Psychology: Animal Behavior Processes, 7, 109-128.

Blair, C. A. J., \& Hall, G. (2003a). Changes in stimulus salience as a result of stimulus preexposure: Evidence from aversive and appetitive testing procedures. Learning \& Behavior, 31, 185-191.

Blair, C. A. J., \& Hall, G. (2003b). Perceptual learning in flavor aversion: Evidence for learned changes in stimulus effectiveness. Journal of Experimental Psychology: Animal Behavior Processes, 29, 39-48.

Bouton, M. E., \& King, D. A. (1983). Contextual control of extinction of conditioned fear: Tests for the associative value of the context. Journal of Experimental Psychology: Animal Behavior Processes, 9, 248-265.

Bouton, M. E., \& King, D. A. (1986). Effect of context on performance to conditioned stimuli with mixed histories of reinforcement and nonreinforcement. Journal of Experimental Psychology: Animal Behavior Processes, 12, 1-12.

Fanselow, M. S. (1982). The postshock activity burst. Animal Learning \& Behavior, 10, 448-454.

Hall, G. (2003). Learned changes in the sensitivity of stimulus representations: Associative and nonassociative mechanisms. Quarterly Journal of Experimental Psychology: Comparative and Physiological Psychology, 56(B), 43-55.

Hall, G., \& Pearce, J. M. (1982). Changes in stimulus associability during conditioning: Implications for theories of acquisition. In M. L. Commons, R. J. Herrnstein, \& A. R. Wagner (Eds.), Quantitative analyses of behavior, Vol. III: Acquisition (pp. 221-239). Cambridge, MA: Ballinger.

Kasprow, W. J., Schachtman, T. T., Cacheiro, H., \& Miller, R. R. (1984). Extinction does not depend on degradation of event memories. Bulletin of the Psychonomic Society, 22, 95-98.

Kehoe, E. J., Weidemann, G., \& Dartnall, S. (2004). Apparatus exposure produces profound declines in conditioned nictitating-membrane responses to discrete conditioned stimuli by the rabbit (Oryctolagus cuniculus). Journal of Experimental Psychology: Animal Behavior Processes, 30, 259-270. 
Mackintosh, N. J. (1975). A theory of attention: Variation in the associability of stimuli with reinforcement. Psychological Review, 82, 276298.

McLaren, I. P. L., Kaye, H., \& Mackintosh, N. J. (1989). An associative theory of the representation of stimuli: Applications to perceptual learning and latent inhibition. In R. G. M. Morris (Ed.), Parallel distributed processing: Implications for psychology and neurobiology (pp. 102130). Oxford, England: Clarendon Press.

Mondragón, E., \& Hall, G. (2002). Analysis of the perceptual learning effect in flavour aversion learning: Evidence for stimulus differentiation. Quarterly Journal of Experimental Psychology: Comparative and Physiological Psychology, 55(B), 153-169.

Pearce, J. M., \& Hall, G. (1980). A model for Pavlovian learning: Variations in the effectiveness of conditioned but not of unconditioned stimuli. Psychological Review, 87, 532-552.

Pearce, J. M., Kaye, H., \& Hall, G. (1982). Predictive accuracy and stimulus associability: Development of a model of Pavlovian learning. In M. L. Commons, R. J. Herrnstein, \& A. R. Wagner (Eds.), Quantitative analyses of behavior, Vol. III: Acquisition (pp. 241-255). Cambridge, MA: Ballinger.

Randich, A. (1981). The US preexposure phenomenon in the conditioned suppression paradigm: A role for conditioned situational stimuli. Learning and Motivation, 12, 321-341.

Randich, A., \& LoLordo, V. M. (1979). Associative and nonassociative theories of the UCS preexposure phenomenon: Implications for Pavlovian conditioning. Psychological Bulletin, 86, 523-548.

Rescorla, R. A., \& Cunningham, C. L. (1977). The erasure of reinstated fear. Animal Learning \& Behavior, 5, 386-394.

Rescorla, R. A., \& Heth, C. D. (1975). Reinstatement of fear to an extinguished conditional stimulus. Journal of Experimental Psychology: Animal Behavior Processes, 1, 88-96.

Rescorla, R. A., \& Wagner, A. R. (1972). A theory of Pavlovian conditioning: Variations in the effectiveness of reinforcement and nonreinforcement. In A. H. Black \& W. F. Prokasy (Eds.), Classical conditioning II: Current research and theory (pp. 64-99). New York: AppletonCentury-Crofts.

Richards, R. W., \& Sargent, D. M. (1983). The order of presentation of conditioned stimuli during extinction. Animal Learning \& Behavior, 11, $229-236$.

Wagner, A. R. (1981). SOP: A model of automatic memory processing in animal behavior. In N. E. Spear \& R. R. Miller (Eds.), Information processing in animals: Memory mechanisms (pp. 5-47). Hillsdale, NJ: Erlbaum.

Received March 25, 2004 Revision received December 14, 2004 Accepted December 16, 2004

\section{E-Mail Notification of Your Latest Issue Online!}

Would you like to know when the next issue of your favorite APA journal will be available online? This service is now available to you. Sign up at http://watson.apa.org/ notify/ and you will be notified by e-mail when issues of interest to you become available! 